\title{
Animal Model of Depression: Effects of Electroconvulsive Shock Therapy
}

\author{
RICHARD J. KATZ \\ Mental Health Research Institute, Department of Psychiatry \\ University of Michigan Medical Center, Ann Arbor, MI 48109
}

Received 12 May 1980

\begin{abstract}
KATZ, R. J. Animal model of depression: Effects of electroconvulsive shock therapy. NEUROSCI. BIOBEHAV. REV. 5(2) $273-277,1981$. - We have previously identified a potentially accurate and specific behavioral and neuroendocrine model of depression in the rat. The most effective somatic treatment for severe depressions is electroconvulsive shock therapy (ECT). ECT was investigated in the preclinical model using a factorial design, and was found to be a specific and effective treatment both behaviorally and endocrinologically. These results suggest the model may successfully reflect the efficacy not only of pharmacological but additionally of other types of somatic treatments.
\end{abstract}

Activity Ambulation Corticosterone Defecation Depression model Electroconvulsive shock
ECT

IN previous studies we have identified a number of noise stress sensitive behavioral and neuroendocrine responses which may be used to model depression in the rat (e.g., $[5,6])$. The model, based upon the reduction of acute stress induced activation after a history of chronic stress appears pharmacologically specific, at least in preliminary tests. Although further tests are necessary, heretofore only clinically effective agents have reversed the behavioral deficit, and also (generally), an accompanying pituitary-adrenal hyperactivity.

Another effective treatment for depression, and indeed the treatment most typically employed for drug-refractory cases is electroconvulsive shock therapy $[1,4]$. To date the only somatic treatments to have been evaluated in the depression model have been drugs. The present report therefore examined the efficacy of a non-pharmacological treatment, ECT, in the depression model.

\section{METHOD}

\section{Subjects}

Forty-eight adult male Sprague-Dawley rats (Charles River Farms), each seventy days at the start of testing, were housed two rats/cage in standard $25 \times 18 \times 17 \mathrm{~cm}$ rack mounted cages, with food (Teklad $4.0 \%$ fat rodent diet S-0836) and tap water continuously owailable. Day/night cycles of $12 \mathrm{hr} / 12 \mathrm{hr}$ (lights on $=0700-1900 \mathrm{hr}$ ) were automatically programmed.

\section{Apparatus and Behavioral Procedure}

Rats were tested in a standard $1.22 \mathrm{~m}^{2} \times 45 \mathrm{~cm}$ height white Plexiglas open field, as previously described $[5,6]$. Each subject received a single six minute placement in the field for behavioral observation prior to sacrifice. A complete $(2 \times 2 \times 2)$ factorial design was employed, with the factors being: acute stress vs. no acute stress, chronic stress vs. no chronic stress, and ECT vs. control treatment. Acute stress consisted of removal from double housing and 1 hour's exposure to $95 \mathrm{~dB}$ of white noise, as opposed to immediate open field testing under basal conditions. Chronic stress consisted of a regimen of unpredictable aversive stimulation, involving over a 21 day period: exposure to 60 minutes of unpredictable shock (avg. $1 \mathrm{~mA}, 1-10 \mathrm{sec}$ duration; average 1 shock/min, ( 3 exposures)), $40 \mathrm{hr}$ food deprivation ( 2 times), cold swim at $4^{\circ} \mathrm{C}$ for five min ( 3 times), $40 \mathrm{hr}$ water deprivation ( 2 times), 5 min exposure to the heat stress at $40^{\circ} \mathrm{C}(2$ times), 30 min shaker stress (2 times), reversal of day/night cycle ( 2 times). Stressors were delivered in a semirandom fashion every 1-2 days throughout the lighting cycle from $0800 \mathrm{hr}$ through $2200 \mathrm{hr}$. Order of stress administration was identical to previous descriptions $[5,6]$ and is presented in Table 1. The third factor, ECT, was delivered via transpinnate electrodes. Shock consisted of a $0.2 \mathrm{sec}$ train of $400 \mathrm{~V}$ shock at $60 \mathrm{~Hz}$. Shocks were administered seven times, separated from each other by $48 \mathrm{hr}$, and beginning one week after the onset of the chronic stress procedure. Care was taken to separate chronic stress administration and ECT administration by a minimum of $8 \mathrm{hr}$ to minimize the direct influence of amnestic effects upon stress related behavioral changes. Control procedure for electroshock involved all procedures including attachment of electrodes, but shock was not delivered. Testing began $24-48 \mathrm{hr}$ after the end of the chronic stress and ECT treatments.

The following behavioral measures were taken for open field testing: outside squares (min $0-3$ ), inside squares (min 0-6), motor latency (i.e., latency to initial movement, in sec), defecation score (bolus count $/ 6 \mathrm{~min}$ ) and defecation latency

\footnotetext{
${ }^{1}$ Correspondence to Dr. Katz at Department of Psychology, Johns Hopkins University, Charles and 34th Street, Baltimore, MD 21218.
} 
TABLE 1

\begin{aligned} & \hline Day \multicolumn{1}{c}{ Treatment } \\ & \hline 1 Shock \\ & 2 Food deprivation $(40 \mathrm{hr}) \\ & 3$ Cold swim \\ & 4 Water deprivation $(40 \mathrm{hr}) \\ & 5 \\ & 6$ Heat stress \\ & 7 Shaker stress \\ & 8 Light/dark cycle reversal \\ & 9 Cold swim \\ & 10 Shock \\ & 11 Switch cage mates \\ & 12 Heat and switch cage mates \\ & 13 Remove food and increase housing density (4/cage) \\ & 14 \\ & 15 Light/dark cycle reversal \\ & 16 Cold swim \\ & 17 \\ & 18 Shock \\ & 19 Water deprivation $(40 \mathrm{hr}) \\ & 20$ Shaker \\ & 21 Test \\ & \hline\end{aligned}

(in sec). A composite activation score based upon sum of relative ranks across the above categories was also computed (see [5,6]). At the close of testing rats were removed for immediate $(<20 \mathrm{sec})$ sacrifice by decapitation, with trunk blood collected in heparinized tubes. Blood was centrifuged at $2400 \mathrm{rpm}$ for $40 \mathrm{~min}$, and plasma was removed and frozen at $-40^{\circ} \mathrm{C}$ for subsequent determination of corticosterone by competitive protein binding assay, using rat corticosterone as the assay standard [8].

Statistical analysis was by univariate analysis of variance, with Sheffe comparisons for the determination of post-hoc significance.

\section{RESULTS}

The present findings suggest that otherwise untreated rats were made hyperactive by acute noise stress exposure. Conversely, defecation measures were typically decreased by noise stress. These effects were both reduced in magnitude by a history of chronic stress, but were restored by ECT. Results are presented below and in Figs. 1-7.

Outside squares activity (Fig. 1) was reliably different across cells, $F(7,40)=11.2, p<0.0001$, and this was due to specific acute noise stress induced increases in control (i.e., non-chronically stressed) rats $(\mathrm{F}=23.8, p<0.0005$; throughout the analysis a post-hoc probability of $p$ less than 0.05 is taken as a criterion of statistical significance. Unless specified to the contrary, all differences to be reported upon were significant at this level) and this increase also was present in chronically stressed ECT treated groups $(\mathrm{F}=24.6)$, in comparison with their respective non-acutely stressed controls. This increase was not present in non-ECT treated chronically stressed or ECT treated non-chronically stressed groups with respect to their control groups (both Fs $<1$ ).

Inside squares activity (Fig. 2) across cells differences

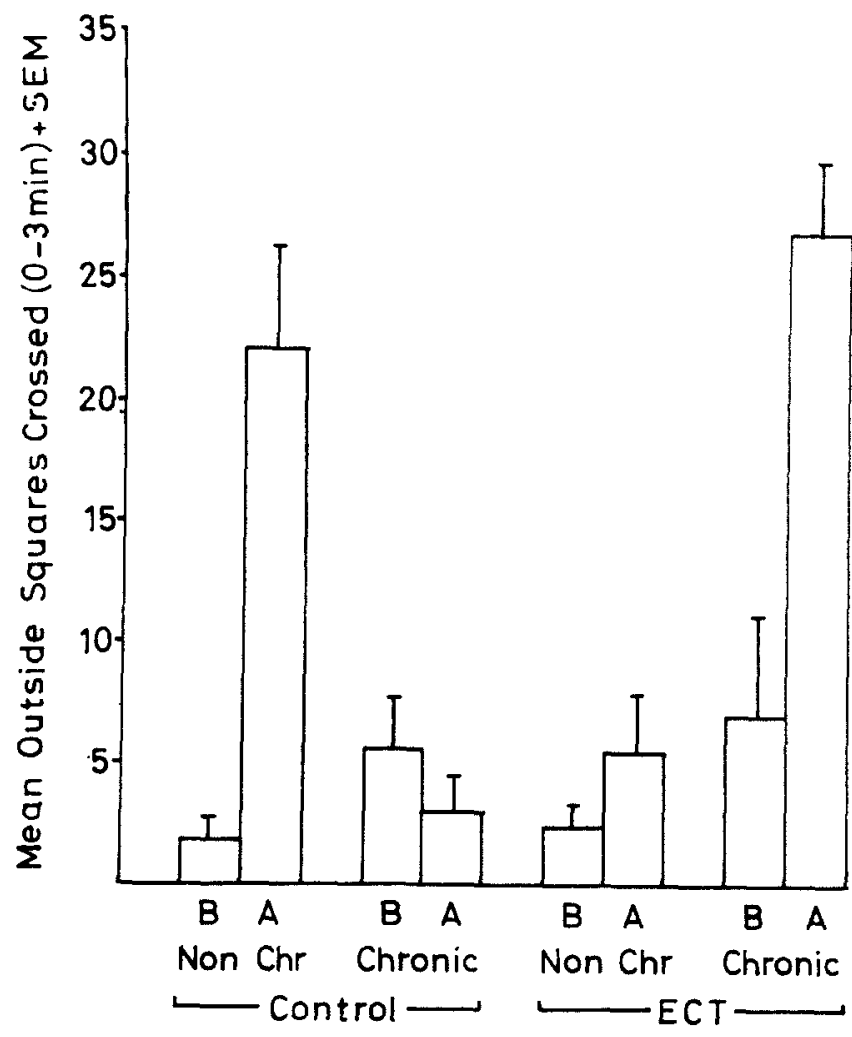

FIG. 1. Mean Motor Activity (outside squares $/ 3 \mathrm{~min}$ ) plus standard error for open field testing of the rat in a factorial design. Factors were basal (B) vs. acutely stressed (A) by $95 \mathrm{~dB}$ white noise; nonchronic (NONCHR) vs. chronic stress regimens (CHRONIC) consisting of 3 weeks exposure to unpredictable stressors and sham (CONTROL) vs. electrocortical shock (ECT) given as a series of seven transpinnate shocks. Rats were individually placed in the field basally or immediately after acute stress; three weeks of chronic stress or control, and seven sessions of ECT or control preceded the acute stress.

were not reliably different from chance $(\mathrm{F}=1.3 \mathrm{df}$ as above, $p=0.3$ ) although those changes which did occur were generally in predicted directions. The absence of any overall reliable differences precluded further analysis.

Motor latency measures (Fig. 3) confirmed the relationship seen for outside squares activity. Latency values differed reliably across cells, $F(7,40)=2.8, p<0.02$, and this was due to reduced latencies brought about by acute stress in otherwise untreated $(F=34.8)$ and in ECT treated, chronically stressed $(F=5.1)$ rats. Acute stress typically elevated latency scores above respective control levels for both chronic stress without ECT, and ECT without chronic stress groups (Fs, respectively $=1.3,0.6$ ) although neither change was reliable beyond chance.

Defecation scores (bolus count; Fig. 4) typically showed patterns converse to the above measures. Cells differed reliably among each other, $\mathrm{F}(7,40)=9.1, p<0.0001$, due to acute noise stress induced decreases in defecation in controls $(F=34.8)$ and in ECT treated chronically stressed rats $(F=4.5)$. Significant reductions in defecation scores were also present in ECT treated non-chronically stressed rats $(F=15.9)$ but not in chronically stressed controls $(F=0.9)$. 


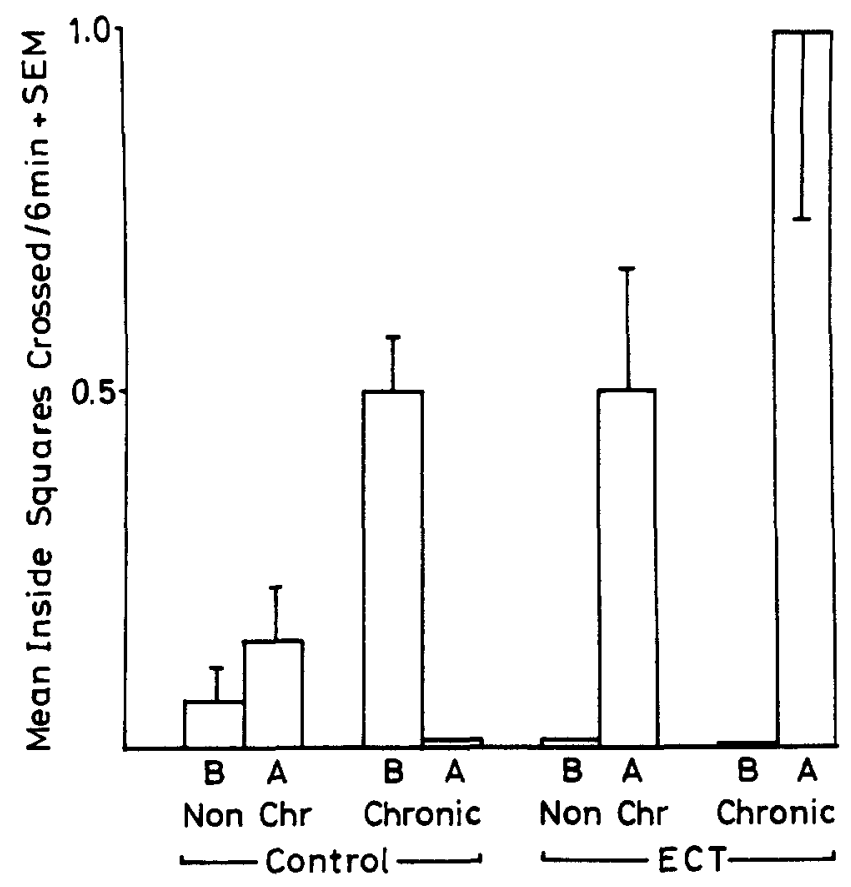

FIG. 2. Mean inside squares traversed per six minutes open field exposure, plus standard error. Figure legend as in Fig. 1.

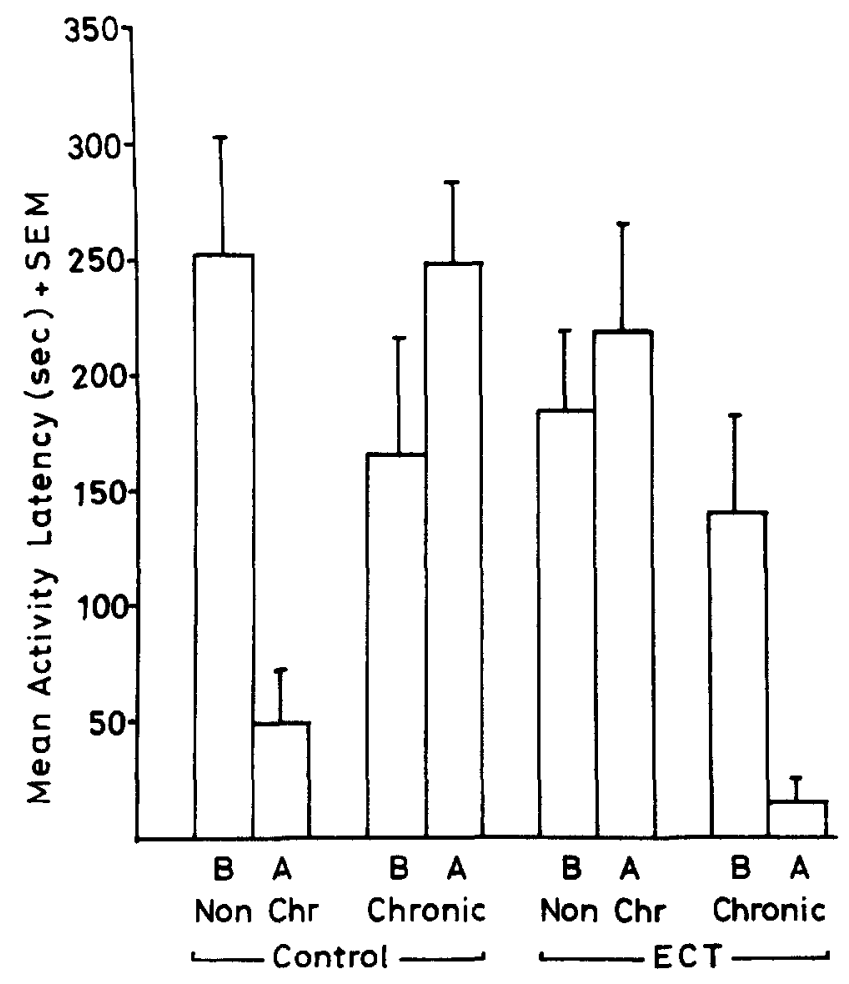

FIG. 3. Mean latency (sec) to initial movement in an open field plus standard error. Figure legend as in Fig. 1.

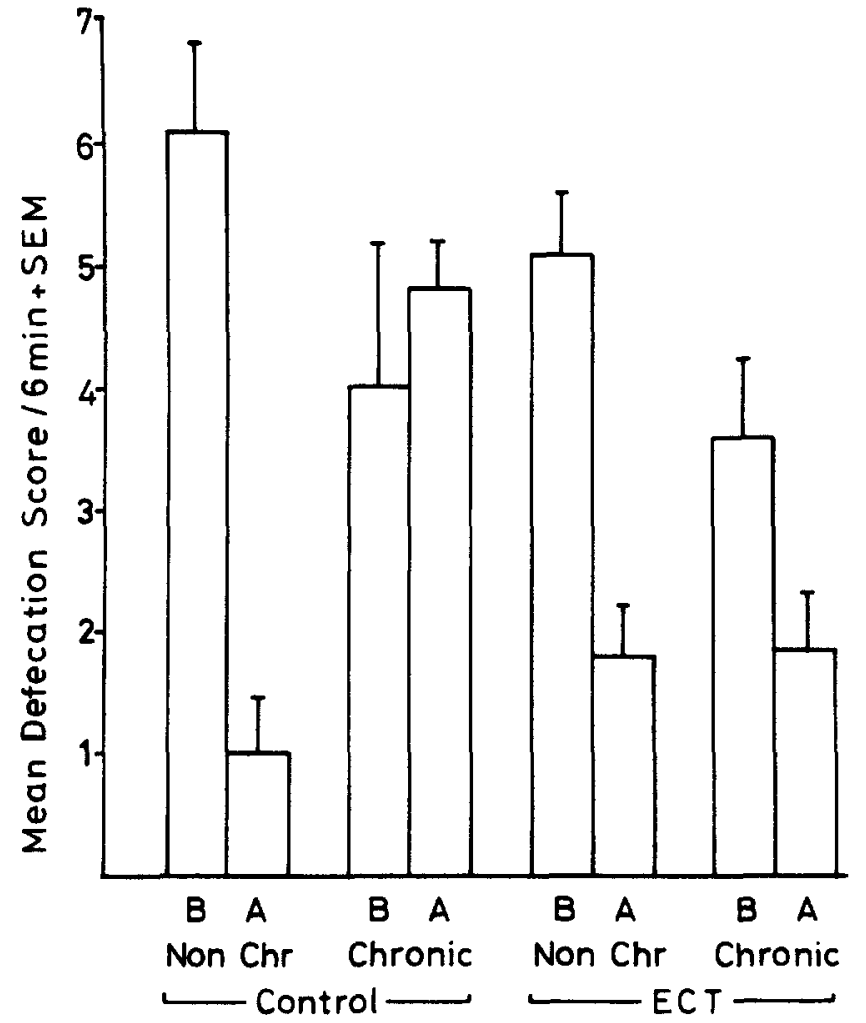

FIG. 4. Mean defecation score (bolus count per six minute open field exposure) plus standard error. Figure legend as in Fig. 1.

Defecation latency (Fig. 5) was quite similar to the above, $\mathbf{F}(7,40)=3.1, p<0.01$. Latencies were increased in control rats by acute noise stress $(F=12.6)$ and similarly increased in ECT treated chronically stressed $(F=3.9)$ and in ECT treated, non-chronically stressed $(F=4.1)$ rats. No such reliable increase was present in chronically stressed, non-ECT treated rats however $(F=0.9)$.

These findings were further reflected in plasma corticosterone values (Fig. 6). Cells were reliably different, $F(7,40)=5.8, p<0.001$, reflecting inter alia acute stress induced elevations of resting corticosterone $(F=19.1)$, and an increase (in comparison with basal rats) in circulating corticosterone due to chronic stress $(F=7.2$, both values in comparison with otherwise basal controls, stress induced increase above basal levels). This was partially reduced by electroshock $(F=4.0)$.

Finally, Fig. 7 presents a composite activity score based upon comparisons of relative ranks of activity across behavioral categories, as described in detail elsewhere [5,6], $F(7,32)=12.5, p<0.0001$. It is evident that based upon an overall measure of open field activity, acute stress increased activity $(F=44.5)$. This was blunted by a history of chronic stress, ( $F=9.6$ for an oppositely signed effect) but was restored by concomitant ECT $(F=9.6)$.

\section{DISCUSSION}

Acute noise stress increased activity in otherwise untreated rats; this activation was reduced by a history of stress, but was restored by a somatic therapy. These behav- 


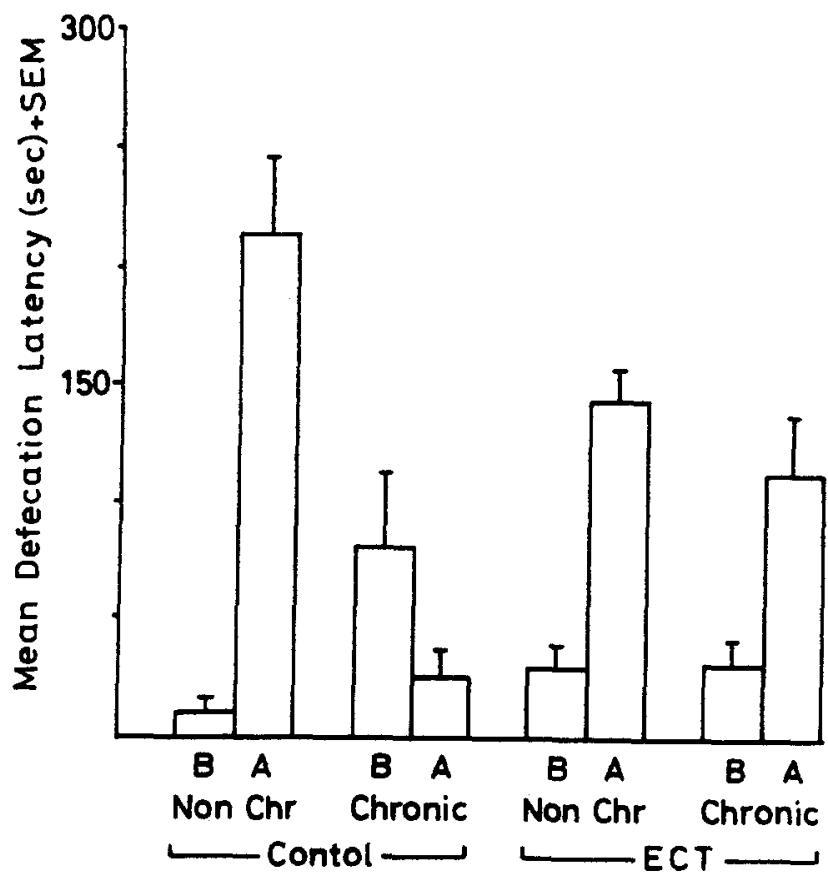

FIG. S. Mean open field defecation htency (sec to initial bolus) plus standard error. Figure legend as in Fig. 1.

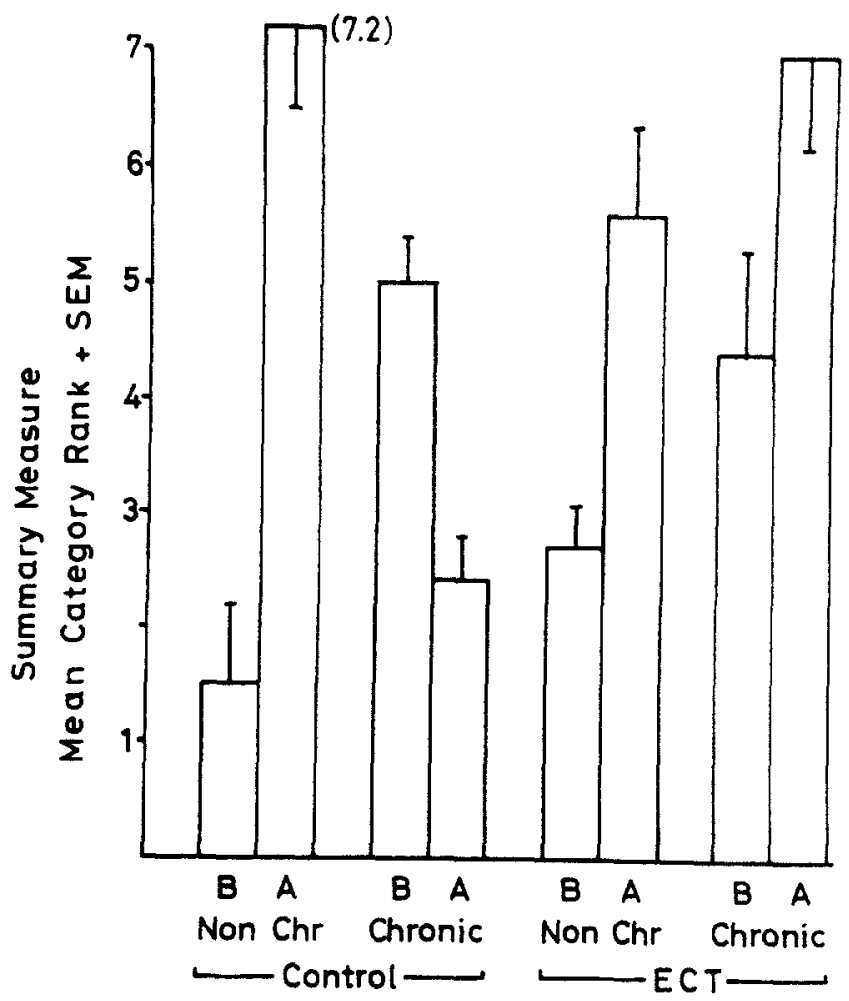

FIG. 7. Mean overall rank and standard error. Composite score across open field ranks as presented in Figs. 1 through 5 . Figure legend as in Fig. 1.

ioral findings were further supported by measures of defecation and also by endocrine measures.

The results replicate the major behavioral changes seen in previous studies of the depression model, and for the first time extend the range of successful therapeutic interventions to a non-pharmacological treatment. A review of previously published studies indicates that ECT reversal of model depressive syndromes is not unique to the present model, nor however is it universal. On the one hand, successful ECT reversal of helplessness and the related behavioral despair model has been reported and replicated $[2,3,9]$. On the other hand, less success has been found after severe isolation in primates [7], although a limited reversal of aberrant behaviors has been reported in the latter case, recovery was at best partial. Conceptually, the present model seems closer to the first class of model than to the second, although some differences do exist. Nonetheless, it would be predicted on grounds of similarity that ECT should be successful, and indeed it was.

Examination of the activity measure (Fig. 1), motor activity latency (Fig. 2), and the summary measure (Fig. 7) indicate that the therapeutic effects of ECT were not merely an algebraic sum of nonspecific forms of behavioral facilitation. ECT baseline measures without acute stress were generally normal. Basal activity was slightly increased after ECT and stress induced activity was decreased. The first finding is dissimilar in comparison with earlier results in a related experiment by Porsolt [9]. The second finding may indicate some form of "cross-tolerance" between electroshock and 
acute noise stress, or possibly that electroshock was the equivalent of a stressor in basal animals, producing, of necessity, a history of stressful experiences. In either case the experimental effects appeared to be related to the reversal of an underlying pathophysiological state induced by experimental means. These findings replicate, supplement and extend previous studies, further supporting the potential utility of the new animal model. As noted, however, still further tests of drugs and other treatments are necessary to further evaluate the model.

\section{ACKNOWLEDGMENTS}

Supported in part by grant MH 31588 from the National Institute of Mental Health (Dr. Bernard J. Carroll, chief investigator). Technical assistance by Giulio Baldrighi and Steven Hirsh, editorial assistance by $A$ be Feingold and statistical assistance by Robert Shea are acknowledged with gratitude. Dr. Katz is a Sloan Foundation Fellow in Neuroscience.

\section{REFERENCES}

1. Abrams, R. and M. Fink. Clinical experiences with multiple electroconvulsive treatments. Comp. Psychiat. 13: 115-121, 1967.

2. Brett, C. W. The generality of learned helplessness theory: Effects of electroconvulsive shock. Doctoral dissertation, Virginia Polytechnic Institute, Xerox, University Microfilm 77-22056, 1977.

3. Dorworth, T. R. and J. B. Overmeier. On learned helplessness: The effects of electroconvulsive shocks. Physiol. Psychol. 5: 355-358, 1977.

4. Fink, M. Convulsive Therapy, Theory and Practice. New York: Raven Press, 1979.

5. Katz, R. J., K. A. Roth and B. J. Carroll. Acute and chronic stress effects on open field activity in the rat: Implications for a model of depression. Neurosci. Biobehav. Rev. 5: 247-251, 1981.
6. Katz, R. J., K. A. Roth and K. Schmaltz. Amphetamine and tranylcypromine in an animal model of depression: Pharmacological specificity of the reversal effect. Neurosci. Biobehav. Rev. 5: 259-264, 1981.

7. Lewis, J. K. and W. T. McKinney. Effects of electroconvulsive shock on the behavior of normal and abnormal rhesus monkeys. Behav. Psychiat. 37: 687-693, 1976.

8. Murphy, B. E. P. Some studies of the protein binding of steroids and their application to the routine micro and ultra-micro measurement of various steroids in body fluids by competitive protein binding radio assay. J. clin. Endocr. 27: 973-990, 1967.

9. Porsolt, R. D., G. Anton, N. Blavet and M. Jalfre. Behavioral despair in rats: A new model sensitive to antidepressant treatments. Eur. J. Pharmac. 47: 379-391, 1978. 